\title{
Diseño y validación de un cuestionario sobre percepción de igualdad en el mundo laboral*
}

\author{
Inge Axpe** \\ Ainhoa Subinas*** \\ Ainhoa Berciano****
}

\section{Resumen}

En la actualidad son muchos los trabajos de investigación que demuestran que, desde la perspectiva de género, nos encontramos aún muy lejos de haber alcanzado la igualdad en el ámbito laboral, y que queda aún mucho trabajo por delante para que ésta pueda llegar a ser efectiva. Sin embargo, en nuestro entorno, y especialmente entre la juventud, prevalece una falsa percepción de que dicha igualdad laboral ya ha sido alcanzada. Con el fin de valorar el grado de esta percepción que tienen las y los jóvenes actuales, en el presente artículo mostramos el diseño teórico y validación de un cuestionario que mide las relaciones laborales desde cinco ámbitos distintos: jerarquía, roles y dinámicas grupales, naturalización, adecuación a los tipos de trabajo y líneas estratégicas.

Palabras clave: Igualdad, Trabajo, Naturalización Biológica, Roles de Género, Espacio Público.

* Recibido el 25 de octubre de 2017, aceptado el 22 de enero de 2020.

** Profesora y investigadora del Departamento de Psicología Evolutiva y de la Educación, Universidad del País Vasco/Euskal Herriko Unibertsitatea, Leioa, España. inge.axpe@ehu.eus / https://orcid.org/0000-0002-1048-1595

*** Profesora y investigadora del Departamento de Didáctica de la Matemática y de las Ciencias Experimentales, Universidad del País Vasco/Euskal Herriko Unibertsitatea, Leioa, España. ainhoa.subinas@ehu.eus / https://orcid.org/00000002-6874-5194

**** Profesora y investigadora del Departamento de Didáctica de la Matemática y de las Ciencias Experimentales, Universidad del País Vasco/Euskal Herriko Unibertsitatea, Leioa, España. ainhoa.berciano@ehu.eus / https://orcid.org/00000001-7399-4745 
Design and Validation of a Questionnaire about The Perception of Equality in The World of Work

\begin{abstract}
Many studies show that from a gender perspective we are still a long way from achieving equality in the workplace, and that much work needs to be done before it is attained. However, in our environment, and especially among young people, a false perception prevails that equality in the workplace has already been achieved. To assess the degree of this perception among young people today, this article presents the theoretical design and validation of a questionnaire that measures labor relations considering five different realms: hierarchy, roles and group dynamics, naturalization, and adaptation to types of work and strategic lines.
\end{abstract}

Keywords: Equality, Labor, Biological Naturalization, Gender Roles, Public Space. 


\section{Introducción}

En estos últimos años está surgiendo una nueva preocupación en el ámbito de la Investigación desde la perspectiva de género, debida a la falsa sensación o espejismo de igualdad en la que nuestra sociedad está inmersa y, más en especial, las y los adolescentes. A este respecto, ya en el informe "¿lgualmente? Alumnado y género, percepciones y actitudes" del Ministerio de Trabajo e Inmigración (2010:37) se destaca que:

Respecto a las percepciones generales acerca de la desigualdad y la discriminación por razón de género, aún siendo conscientes de la existencia de dicha desigualdad, en general, se detecta cierta dificultad en las y los jóvenes para visualizar en su conjunto las diferentes formas de discriminación, las conexiones entre ellas (ámbito privado, ámbito doméstico, ámbito público) y su conexión con los roles y estereotipos que ellos mismos reproducen.

Esta realidad social ha impulsado a investigadoras $e$ investigadores a intentar encontrar las razones de esta percepción de falsa igualdad, destacando, entre otras, que en la actualidad vivimos nuevas formas de dominación que muestran cierta tolerancia con la igualdad formal pero que no aceptan la igualdad de funciones o material entre los hombres y las mujeres (SuárezVillegas, 2013). Este hecho, que dificulta la detección del sexismo y por tanto su erradicación, no es extraño, pues para mantener y legitimar la inequidad de género ésta debe ser aceptada y asumida tanto por quienes se benefician directamente de la existencia de estructuras patriarcales (los hombres), como por quienes padecen la desigualdad, esto es, las mujeres (Fraser; Osborne; Sibley, 2015). De este modo, existen estrategias sutiles que constantemente recuerdan la vigencia de un orden «natural» en las relaciones de dominación entre hombres y mujeres (Suárez-Villegas, 2013). Esta "naturalización" de lo social, es decir, de los roles y características, asignados a cada género, dificulta la identificación del sexismo, y perpetúa la subordinación de la mujer, de tal manera que, pese a 
que el discurso formal e igualitario haya calado en la sociedad, la igualdad real dista mucho de ser efectiva. Naturalizar las diferencias y el estatus de dominación-subordinación facilita que las mujeres también integren en su sistema de creencias el machismo, porque, al hacerlo y asumir los roles tradicionales dependientes y sumisos que les adscribe el patriarcado estarían, teóricamente, asegurándose la aceptación y protección dentro de una sociedad machista (Fraser et alii, 2015; Glick; Fiske, 2001).

La inequidad de género que aún persiste en la sociedad se hace patente en todos los ámbitos, incluso en aquellos como el laboral, en el que, a pesar de décadas de igualdad formal, de desarrollar acciones positivas y de posicionar la igualdad como prioridad política, no se ha conseguido materializar este compromiso de la forma esperada, observándose avances lentos y parciales (Alfama, 2015:264). No obstante, el hecho de que la igualdad haya estado dentro de la agenda y objetivos de instituciones y empresas ha contribuido a generar la falsa idea o creencia de que ha sido conseguida. Idea que, pese a los datos objetivos que la cuestionan, no termina de ser desechada para aceptar la realidad de que, también en lo laboral, la inequidad en función de sexo sigue muy vigente y continúa perjudicando a las mujeres.

Efectivamente, en el ámbito laboral las mujeres continúan sufriendo una clara discriminación en prácticamente todas las sociedades (Fraser et alii, 2015). Sin embargo, a nivel de creencias y opiniones, según el Informe OIT-Gallup (2017), en España, hombres y mujeres dicen aceptar el trabajo asalariado de éstas en un $99 \%$ de los casos, por encima de las medias mundial (80\%) y europea (97\%). Además, únicamente el $3 \%$ de los hombres españoles manifiesta que preferiría que su mujer no trabajase fuera de casa, un porcentaje que se sitúa por debajo de Europa (12\%) y de la media mundial (29\%).

No obstante, existe un importante abismo entre dichas opiniones y los datos reales, tanto en la esfera pública como en la privada. Según los datos del Informe Mundial sobre Salarios (Organización Internacional del Trabajo, OIT 2016-2017), la 
discriminación laboral persiste, existiendo una brecha salarial de género significativa. Por horas trabajadas, esta brecha que en el centil más bajo es de casi un $5 \%$, aumenta gradualmente hasta casi un $30 \%$ en el centil más alto, favoreciendo siempre a los hombres.

Además, no se han podido encontrar razones objetivas que justifiquen tal diferencia. Es decir, la brecha no puede ser explicada por factores como la educación, la capacitación, la antigüedad o el tipo de trabajo que, tenidos en cuenta, deberían eliminar tal diferencia salarial $e$ incluso, favorecer ligeramente a las mujeres (OIT, 2016-17).

Al fuerte estrés psíquico y físico que generan las desventajas y discriminación objetivas de un menor sueldo, menor estatus, menores oportunidades laborales y la experiencia subjetiva de estigmatización (Borrel et alii, 2010) se suma la doble jornada laboral de las mujeres, que continúan dedicando más tiempo a las tareas del hogar y a las tareas de cuidado (Guedes, 2016; Saunders, 2016).

Efectivamente, la sociedad patriarcal se basa en el establecimiento de nítidas divisiones entre lo público: la justicia, la autonomía y la razón, y lo privado: lo emocional, lo familiar, lo doméstico, invisibilizado y subordinado el segundo y obviando las relaciones de interdependencia entre ambos (Alfama, 2015). El énfasis y la asociación permanente y exclusiva de la mujer al rol maternal y de cuidado (Suárez-Villegas, 2013) perjudica doblemente su labor profesional, ya que "el trabajador ideal" no incluye a madres ni cuidadoras (Correll; Bernard; Paik, 2007). De hecho, la percepción de jefes y responsables laborales es que las mujeres tienen mayor conflicto familia-trabajo, incluso aunque la situación real no sea así (Hoobler; Wayne; Lemmon, 2009), lo que lleva a la discriminación de las mujeres embarazadas que buscan trabajo (Morgan et alii, 2013) y a una menor promoción de las mujeres que son madres (Stamarski; Hing, 2015).

Estos procesos cotidianos de recreación del privilegio que ocurren en el entorno laboral son reflejo, y a la vez tienen un importante papel, en el sostenimiento de un orden social más amplio. No obstante, como bien señalan Santos y Amâncio (2016), pese a que parte de la población es consciente de la existencia de 
inequidad en el entorno laboral, la meritocracia, el individualismo y las atribuciones e interpretaciones personales de la discriminación son explicaciones recurrentes que impiden realizar un análisis en función de género. De esta manera, se elude cualquier responsabilidad individual en el cambio social, y se perpetúa la asimetría de género (Santos; Amâncio, 2016). Por todo ello, identificar y explicitar estos aspectos resulta fundamental, ya que esta primera toma de conciencia es la que podría posibilitar, más adelante, el trabajar con dichas creencias para tratar de construir concepciones más igualitarias. Concretamente, en este trabajo son cinco las cuestiones o áreas sobre las que se centra el análisis.

\section{Dimensiones a analizar}

\section{1- Desarrollo profesional, poder y jerarquía}

Las creencias, asunciones y valores asumidos en la cultura organizacional proveen a sus miembros con un imaginario colectivo para interpretar y actuar en dicho contexto laboral (Stamarski; Hing, 2015). Así, en una cultura en la que se enfatiza la meritocracia, es fácil caer en el error de considerar que cada persona ocupa y recibe la posición y los resultados que merece (Son Hing et alii, 2011), deduciendo así que los hombres merecen su elevada situación laboral, mientras que las mujeres merecen su estatus subordinado (Castilla; Benard, 2010).

No obstante, los estereotipos relativos a las capacidades, habilidades y roles específicos y complementarios tanto de hombres como de mujeres condicionan laboralmente a las mujeres (Manganelli; Bobbio; Canova, 2012), a las que, en general, se les ofrecen roles que suponen menos retos, responsabilidades, liderazgo y, por tanto, menos oportunidades de entrenamiento y mejora que a los hombres (King et alii, 2012; Glick, 2013), dificultando así su promoción profesional desde la propia organización de recursos humanos, condenando a las mujeres a estancarse en su carrera profesional (Stamarski; Hing, 2015). Estos obstáculos se hacen patentes ya desde el momento de la contratación, algo constatado empíricamente (Moss-Racusin et alii, 2012) cuando se evalúa el 
curriculum de quienes supuestamente solicitan el empleo. Si el candidato se considera un hombre, es valorado significativamente como más competente y adecuado para el puesto, y se le ofrece un salario base más elevado que al mismo currículo cuando pertenece a una candidata mujer. Por tanto, el sexismo en el entorno laboral se materializa en forma de un predominio masculino en el acceso al poder y en la capacidad para obtener recursos, privilegios y oportunidades (Waylen, 2014), lo que conlleva peores condiciones laborales y salario para las mujeres (Moss-Racusin et alii, 2012; Sheltzer; Smith, 2014) incluso aunque éstas superen en número a los hombres en la institución (Chappell; Waylen, 2013).

\section{2- Naturalización de la diferencia}

La ideología de género utiliza las diferencias biológicas entre hombres y mujeres para legitimar, en base a un orden "natural", la inequidad en su acceso a los recursos (Budgeon, 2014). Por supuesto, esta producción y explicación social de la diferencia no es neutral, ya que, mientras hombres y mujeres se perciban como sujetos esencialmente distintos, no esperarán ocupar una posición similar en la jerarquía y estructura social (Risman, 2004:432). Así, las categorías de género y sus características asociadas orientan la acción individual, de manera que las personas, hombres y mujeres, materializan en su quehacer cotidiano aquello que se espera de ellas, siendo por tanto un mecanismo clave que organiza la estructura social (Budgeon, 2014). El problema reside en que las características definitorias de la "esencia femenina" son devaluadas, y asociadas con la pasividad, sumisión y superficialidad (Budgeon, 2014). En el entorno laboral, esto facilita el que las mujeres sean vistas como no aptas para posiciones de liderazgo, se les confiera una menor credibilidad y atención y se consideren más adecuadas para tareas en las que sea preciso ejercer cuidados y relacionarse con otras personas desde la emotividad y no tanto la objetividad y el raciocinio.

Además, naturalizar la inequidad de género y aceptar la "neutralidad" del mérito exime a las personas de su responsabilidad 
individual en el cambio de las relaciones entre sexos, reduciendo el problema a la tradición y la historia casi de un modo determinístico, y culpando a las mujeres por no actuar en una sociedad formalmente- igualitaria (Santos; Amâncio, 2011; 2016).

\section{3- Dinámicas y roles grupales}

Pese a la conciencia de la inequidad de género, determinados contextos y situaciones laborales continúan siendo vistos como un entorno "natural" que corresponde a los hombres, de manera que tanto hombres como mujeres tienden a considerar que las cualidades que precisan las personas para desenvolverse adecuadamente en ellas deben ser aquellas generalmente asociadas a los hombres, por ejemplo inteligencia, pragmatismo, y convicción (Santos; Amâncio, 2010). Así, se "duda" de la capacidad de las mujeres para determinadas profesiones y situaciones que exijan liderazgo, mando, "estrategia"... considerando que ese es el papel y el entorno de los hombres más preocupados por el éxito, la gestión y la legalidad, mientras que a ellas se les confiere capacidad para realizar otras tareas menos valoradas y más relacionadas con su "natural" mundo de las relaciones y el cuidado (Santos; Amâncio; Roux, 2015; Santos; Amâncio, 2016). Es decir, se adscriben rasgos de agencia como competencia, asertividad, confianza, independencia, dominancia, agresividad y ambición a los hombres, asignando, por el contrario, rasgos comunitarios como el ser amable, cuidadora, atenta, expresiva, emotiva, dependiente y sumisa, a las mujeres (Eagly; Karau, 2002; Santos; Amâncio, 2016). Precisamente debido a que las mujeres se asocian a rasgos comunitarios y roles subordinados, cuando tratan de ocupar otras posiciones, por ejemplo, de liderazgo, y muestran competencia y confianza en sus capacidades, tienden a ser peor consideradas: menos agradables, con menos habilidades sociales, y menos contratables en comparación con hombres con igual actitud (Rudman et alii, 2012). 


\section{4- Tipología laboral}

La división y adscripción de distintos rasgos y comportamientos "adecuados" para hombres y mujeres es muy patente en los roles y dinámicas grupales dentro de una misma profesión, pero también en la elección y la facilidad o dificultad de acceso a determinadas profesiones, ya que se continúa asociando en mayor medida a los hombres con roles profesionales y a las mujeres con roles familiares (Nosek Banaji; Greenwald, 2002; Santos; Amâncio, 2016). De este modo, aquellas tareas que en su momento fueron relegadas al espacio doméstico y familiar siguen asignándose, aún en el espacio profesional, a las mujeres (Araujo, 2016). Esta creencia respecto a habilidades y características "femeninas o masculinas" tiene un efecto devastador en las mujeres, que resultan perjudicadas cuando acceden al entorno laboral, en mayor medida aún si tratan de acceder a profesiones, posiciones o roles "típicamente" masculinos (Rudman et alii, 2012).

A todas estas dificultades para el acceso al entorno laboral que sufren las mujeres se les suma el desigual reparto en las responsabilidades domésticas y familiares (Blofield; MartínezFranzoni, 2014), lo que determina las decisiones respecto al desarrollo profesional en las parejas heterosexuales, limitando, en general, la carrera de ellas frente a la de sus parejas masculinas (Stamarski; Hing, 2015). Es decir, las responsabilidades familiares y domésticas muchas veces constituyen serios obstáculos para el mantenimiento de una jornada laboral completa, por lo que fuerzan a la interrupción de la carrera profesional para dedicarse a la crianza y relegan a muchas mujeres al entorno familiar y privado (Blofield; Madalozzo, 2013). Esta elección de las mujeres se produce en un entorno fuertemente condicionado que les obliga a adoptar modelos patriarcales tradicionales. Sin embargo, dichas elecciones y relegación al ámbito privados se presentan socialmente como "naturales" (Santamaría, 2013), sin tener en cuenta que "en el patriarcado y en el capitalismo, las prácticas, estereotipos, acciones y condiciones inciden en las decisiones de las mujeres y los hombres" (Cordero, 2017:24). 
5- Líneas estratégicas de gobierno

Las acciones positivas tienen un gran potencial para combatir la inequidad laboral (Fraser et alii, 2015). Estas acciones positivas o líneas estratégicas pueden clasificarse en función de tres categorías: secuenciales, aquellas que aseguran que la asunción de la crianza no perjudique la seguridad laboral (ej: "bajas" o permisos por maternidad y paternidad para el cuidado de hijos/as, jornadas flexibles...), políticas que relevan a la familia en los cuidados (ej: escolarización infantil, programas extraescolares...) o políticas que regularizan y formalizan el cuidado y labor doméstica (Blofield; Martínez-Franzoni, 2014). A pesar de que este tipo de medidas y estrategias tratan de eliminar parte de los obstáculos que encuentran las mujeres para el acceso y mantenimiento en el ámbito laboral, no han sido suficientes para lograr la equidad, calculándose que aún serían precisas al menos 8 décadas para alcanzarla (World Economic Forum, 2014). Además, las acciones positivas pueden resultar muy controvertidas, como es el caso de la adopción de cuotas de género, una medida objeto de fuertes críticas, división social y controversia (Santos; Amâncio, 2010) por violar la norma implícita de la meritocracia que, en el contexto laboral, simbólicamente asignado a los hombres, se atribuye de manera "natural" a éstos (Santos; Amâncio, 2016). De este modo, el efecto de las medidas positivas puede volverse perjudicial en función de cómo sean interpretadas por el entorno, ya que no existe mejor herramienta para continuar restringiendo y sometiendo a un grupo minoritario que el uso de recursos y acciones paternalistas que, de manera encubierta, restringen sus oportunidades (Jackman, 1994). Así, pese a que las acciones positivas no tienen per se un cariz paternalista, podrían ser interpretadas como tal: una ayuda y protección necesarias hacia las mujeres que, de lo contrario, serían incapaces de tener éxito por sí mismas en un entorno "no familiar" para ellas como el laboral (Fraser et alii, 2015). Esta interpretación contribuiría a mantener la jerarquía social, al facilitar que los logros de las mujeres se atribuyan a las acciones positivas en lugar de a su propia capacidad (Fraser et alii, 2015). Todo ello a pesar de que se 
ha demostrado empíricamente que incluso quienes logran su puesto a través de cuotas de género no difieren significativamente de otros candidatos en lo que a competencias y formación se refiere (Allen; Cutts; Campbell, 2016).

\section{Metodología}

Objetivos de la investigación

El actual artículo se enmarca dentro de una investigación más amplia cuyo objetivo principal es evaluar y analizar la percepción actual del alumnado universitario sobre aspectos relacionados con la posible Igualdad en el Mundo laboral desde una perspectiva de género, con el fin de posteriormente diseñar acciones que fomenten la igualdad real.

Para tal fin, nuestro primer paso ha sido la creación de un test que, teniendo en cuenta la situación actual, midiera la percepción del alumnado, teniendo, por tanto, como objetivo principal de este artículo el evaluar la fiabilidad y validez de un test específicamente diseñado para medir la percepción del alumnado universitario.

Participantes

El estudio se ha llevado a cabo con alumnado universitario de la Facultad de Educación de Bilbao cursando primer año del Grado de Educación Primaria. Participaron 186 estudiantes, 129 mujeres (69.35\%) y 57 hombres (30.65\%), con una media de 19.1 años y una $\mathrm{DT}=2.09$ años.

Variables e instrumento de medida

Para el diseño del test se tuvieron en cuenta los 5 ámbitos definidos en el marco teórico de manera generalizada: 1) desarrollo profesional, poder y jerarquía; 2) naturalización de la diferencia; 3) dinámicas y roles grupales; 4) tipología laboral y 5) líneas estratégicas de gobierno. 
En una primera fase se creó un cuestionario piloto que fue remitido a 5 personas expertas en el área para su posterior valoración, de modo que determinaran su idoneidad en tanto a la claridad como a la relación con el tema investigado. Teniendo en cuenta las respuestas obtenidas por parte de las expertas, se replanteó el enunciado de ciertos ítems y se elaboró un test piloto con un total de 21 cuestiones.

En este test piloto todos los ítems se deben responder acorde al grado de conformidad en una escala Likert de 1 a 6 , donde 1 significa totalmente en desacuerdo; 2 desacuerdo; 3 poco en desacuerdo; 4 un poco de acuerdo; 5 de acuerdo y 6 totalmente de acuerdo. A continuación, presentamos el test piloto junto a los ámbitos teóricos asociados inicialmente. 
Tabla 1. Test piloto

\begin{tabular}{|c|c|c|}
\hline $\mathrm{N}^{\mathrm{O}}$ & Texto ítem & Ámbito teórico \\
\hline 1 & $\begin{array}{l}\text { El que en algunas profesiones predominen los hombres (mineros, ingenieros, policías...) y } \\
\text { en otras las mujeres (enfermeras, azafatas, secretarias...) se debe a diferencias naturales } \\
\text { entre ambos sexos. }\end{array}$ & 4 \\
\hline 2 & $\begin{array}{l}\text { Utilizar un lenguaje inclusivo (ej.: los/as ciudadanos/as...) es innecesario ya que el masculino } \\
\text { visibiliza e incluye también a las mujeres. }\end{array}$ & 5 \\
\hline 3 & $\begin{array}{l}\text { Las diferencias en el sueldo de hombres y mujeres de la misma categoría y nivel profesional } \\
\text { responden a diferente nivel de productividad o de capacidad de cada uno/a. }\end{array}$ & 4 \\
\hline 4 & $\begin{array}{l}\text { Que hoy en día te contraten depende únicamente de tu formación, experiencia previa y } \\
\text { capacidades, no de tu sexo. }\end{array}$ & 1 \\
\hline 5 & $\begin{array}{l}\text { Las acciones positivas hacia las mujeres (destinarles ayudas específicas, fomentar su } \\
\text { contratación) perjudican a los hombres y a las propias mujeres, porque les hace parecer } \\
\text { inferiores. }\end{array}$ & 5 \\
\hline 6 & $\begin{array}{l}\text { El que haya más mujeres en profesiones de cuidado (enfermeras, maestras...) y de limpieza } \\
\text { se debe a gustos y tendencias naturales de las mujeres. }\end{array}$ & 2 \\
\hline 7 & Hay más hombres empresarios y políticos porque a ellos les interesa más el poder. & 2 \\
\hline 8 & Un jefe hombre es más objetivo e imparcial y, por tanto, más resolutivo que una jefa. & 1 \\
\hline 9 & $\begin{array}{l}\text { Los hombres llegan más lejos y a mejores puestos en su carrera profesional porque, por } \\
\text { naturaleza, las mujeres son menos competitivas y emprendedoras. }\end{array}$ & 1 \\
\hline 10 & $\begin{array}{l}\text { En el trabajo, los hombres suelen tomar mejores decisiones, porque son más racionales y } \\
\text { objetivos que las mujeres. }\end{array}$ & 1 \\
\hline 11 & $\begin{array}{l}\text { En el espacio público (reuniones laborales, charlas), las mujeres tienen menos } \\
\text { protagonismo, porque les gusta ser más discretas. }\end{array}$ & 3 \\
\hline 12 & $\begin{array}{l}\text { En grupos de trabajo mixtos, se suele escuchar con más detalle cuando interviene un } \\
\text { hombre que una mujer, porque normalmente se explican más claramente. }\end{array}$ & 3 \\
\hline 13 & $\begin{array}{l}\text { Si en una pareja heterosexual hay que encargarse del cuidado de hijos/as u otro familiar, y } \\
\text { alguno de los dos miembros tiene que dejar su trabajo profesional, les gusta más hacerlo a } \\
\text { las mujeres. }\end{array}$ & 3 \\
\hline 14 & $\begin{array}{l}\text { Los hombres son más productivos y rentables para cualquier empresa, ya que las mujeres } \\
\text { suponen muchos más problemas: bajas por maternidad, permisos para cuidado, ausencias } \\
\text { por enfermedades de los hijos/as... }\end{array}$ & 2 \\
\hline 15 & $\begin{array}{l}\text { En estos momentos, las reivindicaciones feministas respecto a condiciones laborales, abusos } \\
\text { de poder etc. han perdido todo su sentido. }\end{array}$ & 5 \\
\hline 16 & $\begin{array}{l}\text { Las bajas de paternidad sólo sirven para que los hombres tengan más vacaciones, ya que } \\
\text { en el momento en que nace el hijo/a el cuidado principal corresponde por naturaleza a la } \\
\text { madre. }\end{array}$ & 3 \\
\hline 17 & $\begin{array}{l}\text { Para igualar las condiciones laborales, estaría bien igualar la duración de los permisos por } \\
\text { paternidad y maternidad para el cuidado del bebé recién nacido. }\end{array}$ & 5 \\
\hline 18 & $\begin{array}{l}\text { En Educación, las mujeres son mayoritarias en niveles de Infantil porque son más cariñosas, } \\
\text { afectivas, cuidadosas... }\end{array}$ & 2 \\
\hline 19 & $\begin{array}{l}\text { En Educación, en niveles superiores como el instituto, como se necesita más disciplina, } \\
\text { aumenta el número de profesores hombres. }\end{array}$ & 2 \\
\hline 20 & $\begin{array}{l}\text { Las mujeres tienden a escoger profesiones y estudios referidos al cuidado (como psicología, } \\
\text { enfermería,...) mientras que los hombres escogen profesiones técnicas porque es lo que la } \\
\text { sociedad espera. }\end{array}$ & 4 \\
\hline 21 & $\begin{array}{l}\text { Las diferencias en la ropa y vestimenta profesionales (ej: faldas en mujeres, uso de tacón, } \\
\text { maquillaje...) se deben a que ellas son más coquetas y les gusta más cuidar la apariencia. }\end{array}$ & 2 \\
\hline
\end{tabular}




\section{Procedimiento}

La administración del test piloto tuvo lugar a lo largo del curso 2016/2017, participando voluntariamente 186 personas de un total de 200 estudiantes de primero del Grado de Educación Primaria (93\%). De manera previa a la administración de la prueba, las personas participantes recibieron información acerca de los objetivos de la investigación y de la voluntariedad de la misma, así como de la posibilidad de abandonar la cumplimentación del cuestionario en cualquier momento del proceso.

El cuestionario se respondió de modo individual y anónimo en un aula informática de la Universidad, mediante una aplicación de encuesta on-line. La cumplimentación se desarrolló en presencia de la profesora responsable del curso correspondiente, variando el tiempo necesario para completar la prueba entre 20-30 min. Se recogieron datos sociodemográficos solicitando al alumnado que rellenara los apartados referidos a edad, sexo, carrera, curso y, de modo automático, fecha de realización del test.

\section{Análisis Exploratorio del Test}

\section{Validación del cuestionario}

El cuestionario, creado acorde a los ámbitos descritos en el marco teórico, fue evaluado por 5 personas expertas en el área. La evaluación realizada por las expertas, en general, mostró un alto grado de conformidad con la redacción y selección inicial de los ítems. En cinco casos se sugirió cierta modificación en la manera en la que estaba escrita o planteada la pregunta, aportaciones que se incorporaron a la prueba piloto del cuestionario. Por otro lado, todas las expertas mostraron la idoneidad de la elección de la escala Likert.

Análisis de la fiabilidad del cuestionario piloto

En primer lugar, se ha medido la fiabilidad interna de la escala en su conjunto, haciendo uso del Test de consistencia interna Alfa 
de Cronbach (.771). A pesar de que dicho índice alcanza un valor aceptable, al evaluar el índice de homogeneidad de los ítems y el alfa de Cronbach en caso de suprimirlos (ver tabla 2), se observa que la eliminación de los ítems 4, 17 y 20 permitiría mejorar el índice de fiabilidad de la escala.

Tabla 2. Índice de homogeneidad y alfa de Cronbach según ítem

\begin{tabular}{lcc}
\hline Test piloto & $\begin{array}{c}\text { Índice de } \\
\text { homogeneidad }\end{array}$ & $\begin{array}{c}\text { Alfa de Cronbach si el elemento se ha } \\
\text { suprimido }\end{array}$ \\
\hline Test piloto: T1 & .331 & .764 \\
Test piloto: T2 & .253 & .770 \\
Test piloto: T3 & .299 & .765 \\
Test piloto: T4 & .107 & .782 \\
Test piloto: T5 & .218 & .770 \\
Test piloto: T6 & .561 & .746 \\
Test piloto: T7 & .521 & .748 \\
Test piloto: T8 & .378 & .763 \\
Test piloto: T9 & .407 & .763 \\
Test piloto: T10 & .495 & .761 \\
Test piloto: T11 & .447 & .759 \\
Test piloto: T12 & .213 & .768 \\
Test piloto: T13 & .384 & .759 \\
Test piloto: T14 & .402 & .758 \\
Test piloto: T15 & .447 & .756 \\
Test piloto: T16 & .436 & .759 \\
Test piloto: T17 & .113 & .784 \\
Test piloto: T18 & .564 & .743 \\
Test piloto: T19 & .519 & .751 \\
Test piloto: T20 & .130 & .782 \\
Test piloto: T21 & .517 & .748 \\
\hline
\end{tabular}

Se procede entonces a suprimir los ítems 4, 17, y 20, obteniendo un alfa de Cronbach de .813 .

Eliminados estos tres ítems, un nuevo análisis ítem a ítem teniendo en cuenta su homogeneidad y el alfa en caso de suprimirlo, revela que la eliminación del ítem 2 , con un índice de homogeneidad bajo (.227), mejoraría el resultado del test, elevando su consistencia interna hasta 822 .

Así, la escala final queda constituida por 17 ítems (ver tabla 3). 
Tabla 3. Estadísticos descriptivos de los 17 ítems resultantes

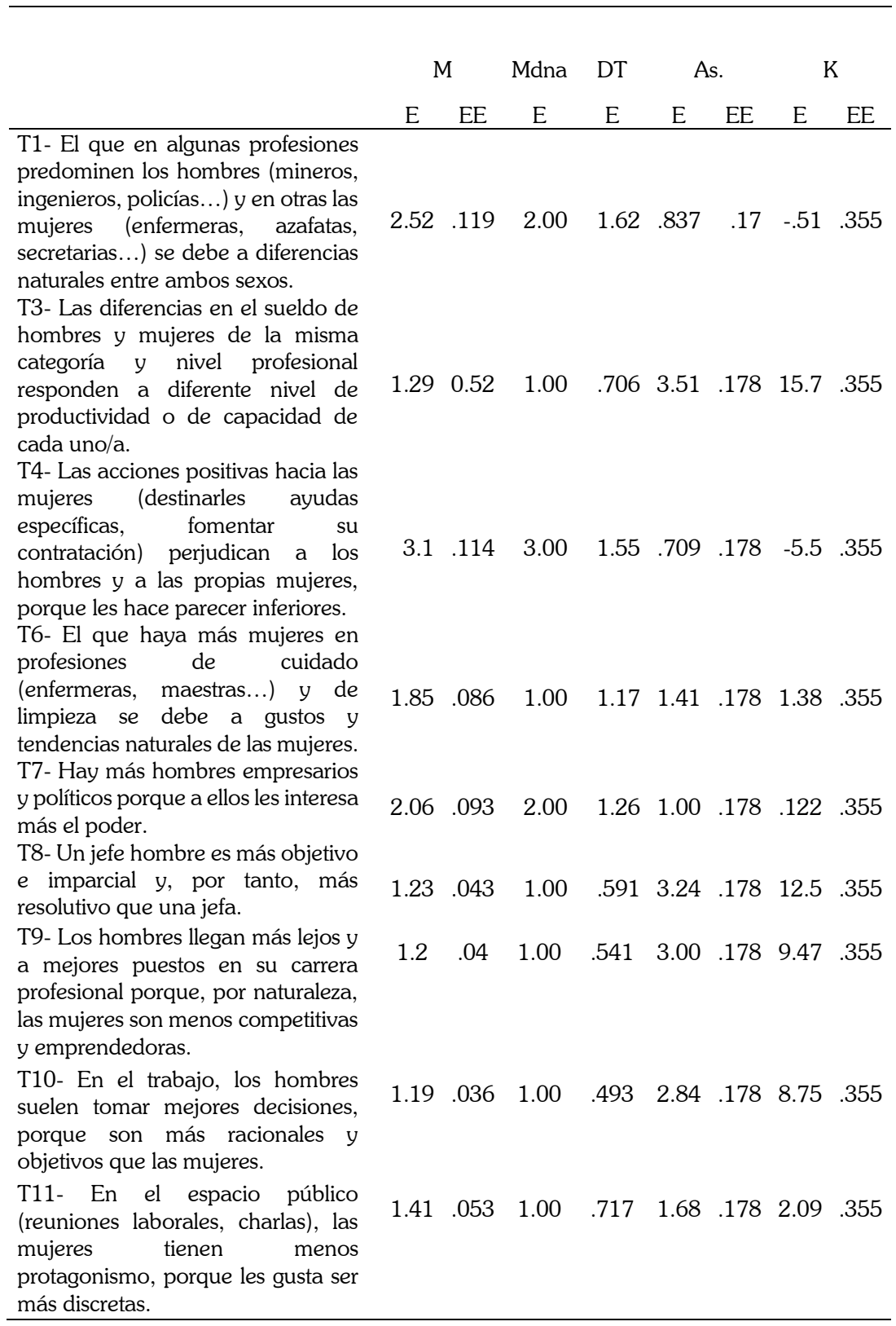


T12- En grupos de trabajo mixtos, se suele escuchar con más detalle cuando interviene un hombre que una mujer, porque normalmente se explican más claramente.

T13- Si en una pareja heterosexual hay que encargarse del cuidado de hijos/as u otro familiar, y alguno de los dos miembros tiene que dejar su trabajo profesional, les gusta más hacerlo a las mujeres.

T14- Los hombres son más productivos y rentables para cualquier empresa, ya que las mujeres suponen muchos más problemas: bajas por maternidad, permisos para cuidado, ausencias por enfermedades de los hijos/as... T15- En estos momentos, las reivindicaciones feministas respecto a condiciones laborales, abusos de poder etc. han perdido todo su sentido.

T16- Las bajas de paternidad sólo sirven para que los hombres tengan más vacaciones, ya que en el momento en que nace el hijo/a el cuidado principal corresponde por naturaleza a la madre.

T18- En Educación, las mujeres son mayoritarias en niveles de Infantil porque son más cariñosas, afectivas, cuidadosas...

T19- Las mujeres tienden a escoger profesiones y estudios referidos al cuidado (como psicología, enfermería,...) mientras que los hombres escogen profesiones técnicas porque es lo que la sociedad espera.

T21- Las diferencias en la ropa y vestimenta profesionales (ej: faldas en mujeres, uso de tacón, maquillaje...) se deben a que ellas son más coquetas y les gusta más cuidar la apariencia. $\begin{array}{llllllll}1.28 & .051 & 1.00 & .695 & 3.16 & .178 & 11.2 & .355\end{array}$

$\begin{array}{llllllll}1.55 & .068 & 1.00 & .924 & 1.80 & .178 & 3.18 & .355\end{array}$

$\begin{array}{llllllll}1.62 & 076 & 1.00 & 1.034 & 1.96 & .178 & 3.94 & .355\end{array}$

$\begin{array}{llllllll}1.67 & .065 & 1.00 & .892 & 1.40 & .178 & 1.69 & .355\end{array}$

$\begin{array}{llllllll}1.33 & .056 & 1.00 & .762 & 2.98 & .178 & 11.0 & .355\end{array}$

$\begin{array}{llllllll}2.25 & .10 & 2.00 & 1.365 & 1.02 & .178 & .208 & .355\end{array}$

$\begin{array}{llllllll}1.62 & .071 & 1.00 & .964 & 1.78 & .178 & 3.23 & .355\end{array}$

$\begin{array}{llllllll}1.88 & .091 & 1.00 & 1.24 & 1.39 & .178 & 1.10 & .355\end{array}$ 
Análisis factorial exploratorio del test final

Para el análisis de la validez estructural, en primer lugar, se ha analizado el índice Kaiser-Meyer-Olkin de adecuación muestral, KMO $(0.839>0.5)$ y el test de esfericidad de Barttet $(0<0.01)$. Ambos valores nos permiten garantizar la adecuación de continuar con el análisis factorial. En particular, se ha realizado un análisis factorial exploratorio sobre la matriz de correlaciones policóricas por el método de ejes principales con rotación varimax, debido a la baja-media correlación hallada entre los factores extraídos. Éste ha proporcionado cinco factores de primer orden que explican un $58.78 \%$ de la varianza.

Estructura del cuestionario final acorde al marco teórico

El análisis de las saturaciones de cada ítem (ver tabla 4), permite reconocer, en la estructura de 5 factores, las dimensiones previamente señaladas en la teoría. 
Tabla 4. Índice de homogeneidad y alfa de Cronbach según ítem

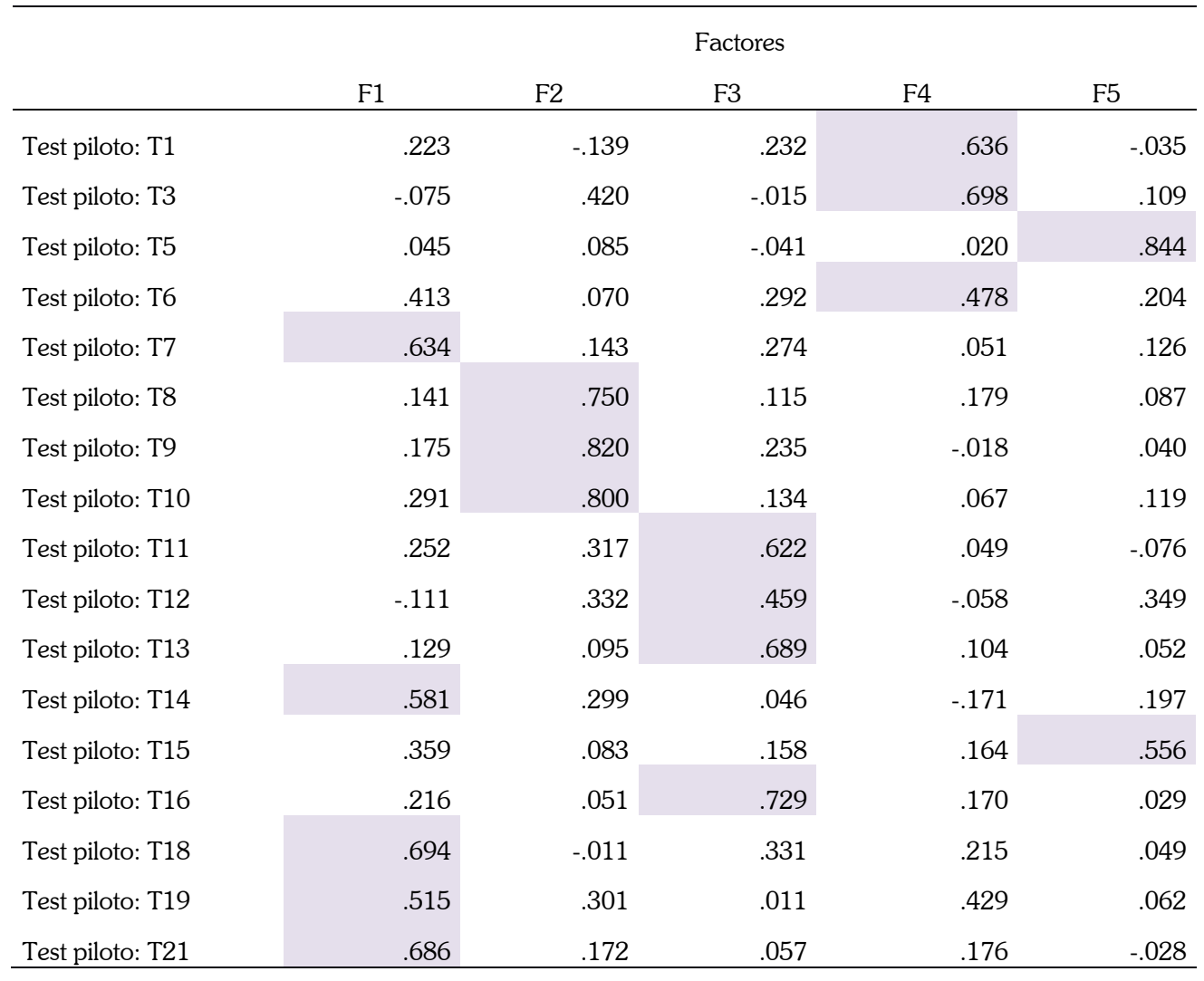

De este modo, el test final formado por los 17 ítems muestra un índice de fiabilidad interna de 0.822 (alfa de Cronbach), en el que los ítems quedan estructurados acorde a las dimensiones planteadas de modo teórico, lo que corrobora la validez del cuestionario (ver tabla 5). 
Tabla 5. Dimensiones y alfa de Cronbach de cada dimensión

\begin{tabular}{clll}
\hline $\begin{array}{c}\text { Componente } \\
\mathrm{N}^{\circ}\end{array}$ & \multicolumn{1}{c}{ Campo Teórico } & Ítems Cuestionario & Alfa de Cronbach \\
\hline F2 & Poder y jerarquía & $8,9,10$ & 0.824 \\
F1 & Naturalización de la diferencia & $7,14,18,19,21$ & 0.739 \\
F3 & Dinámicas y roles grupales & $11,12,13,16$ & 0.625 \\
F4 & Tipos de trabajo & $1,3,6$ & 0.455 \\
F5 & Líneas estratégicas de gobierno & 5,15 & 0.502 \\
\hline
\end{tabular}

\section{Discusión y Conclusiones}

Acorde a los resultados obtenidos, podemos afirmar que el cuestionario diseñado cumple con las condiciones mínimas necesarias para garantizar la fiabilidad y validez del test, lo que permitirá conocer en mayor medida la percepción que tiene el alumnado acerca de las relaciones y nexos implícitos y explícitos que se crean en el mundo laboral desde una perspectiva de género.

Debemos destacar el alto índice obtenido en las subdimensiones Poder y jerarquía y Naturalización, al igual que un buen índice para la dimensión Dinámicas y roles grupales. Aun así, queda trabajo por hacer en lo referente a las dimensiones Tipos de trabajo y Líneas estratégicas, con el fin de poder analizar las razones de sus índices obtenidos.

Una explicación a considerar es, precisamente, lo complejo e interrelacionado de los componentes y dimensiones, pudiendo situarse ciertos ítems en una o más de los factores. Por ello, resultaría conveniente la revisión y mejora de la redacción de dichas cuestiones, cuya distribución o clasificación teórica inicial ha resultado diferente a la esperada en los análisis estadísticos. Precisamente, los ítems que finalmente constituyen la escala Tipos de trabajo $(1,3,6)$, en la que también se incluía el ítem 20 (Las mujeres tienden a escoger profesiones y estudios referidos al cuidado, como psicología, enfermería..., mientras que los hombres escogen profesiones técnicas porque es lo que la sociedad espera) eliminado para mejorar la fiabilidad de la escala, podrían interpretarse como elementos bien de la dimensión Naturalización 
(ítem 1,6$)$ o de la dimensión Jerarquía y poder (ítem 3). No obstante, los resultados muestran una mayor saturación en el factor de Tipos de trabajo, por lo que debería considerarse la posibilidad de revisar la redacción de tales ítems y quizá la inclusión de alguno nuevo para mejorar los índices de tal subescala.

En lo que respecta a la dimensión Líneas estratégicas, destaca el hecho de que, del total de 4 ítems eliminados, dos, ítem 2 y 17 , ("Utilizar un lenguaje inclusivo, ej.: los/as ciudadanos/as..., es innecesario ya que el masculino visibiliza e incluye también a las mujeres" y "Para igualar las condiciones laborales, estaría bien igualar la duración de las bajas por paternidad y maternidad") correspondían a esta dimensión. Es posible que el ítem 2 no guardase una relación directa con el entorno laboral, y que el ítem 17 aún resulte una situación demasiado lejana y desconocida para alumnado de 19 años, dificultando su conciencia respecto a la carga $\mathrm{y}$ las trabas que un reparto desigual de las tareas $\mathrm{y}$ responsabilidades familiares y domésticas supone para el desarrollo profesional de las mujeres (Blofield; Martínez-Franzoni; 2014; Stamarski; Hing, 2015).

El peor resultado de la dimensión de Líneas estratégicas podría estar apuntando hacia esa falacia o falsa creencia tan extendida de que la igualdad entre hombres y mujeres ya existe; pese a que realmente diste mucho de haberse alcanzado (World Economic Forum, 2014) y, por lo tanto, que el alumnado realmente considerase innecesaria la puesta en práctica de medidas positivas hacia las mujeres.

Como limitación del estudio deberíamos destacar el tamaño y tipología muestral elegidos, éstos no han sido de modo aleatorio y, por tanto, podrían obtenerse ciertas variaciones en los análisis.

No obstante, las 5 dimensiones teóricas planteadas parecen, en vista de los resultados, corroborarse, aun cuando debería tenerse en cuenta la revisión de ciertos enunciados y quizá la inclusión de nuevas preguntas para mejorar los índices de algunas de las subescalas.

En cualquier caso, la relevancia de la temática impulsa a continuar investigando en las creencias y percepciones de la 
juventud respecto a la igualdad entre sexos. Algo especialmente importante en un ámbito como el laboral, en el que los datos empíricos pueden, con facilidad, ayudar a desmontar los mitos y creencias tan instauradas en la sociedad respecto al reparto de espacios, roles y características "propias y naturales" de cada sexo, y que se siguen utilizando, aún en el s. XXI, para justificar y mantener un desigual reparto del poder, bienestar y riqueza entre hombres y mujeres.

\section{Referencias bibliográficas}

Allen, Peter; CutTS, David; CAMPBell, Rosie. Measuring the quality of politicians elected by gender quotas: Are they any different? Political Studies (64), 2016, pp.143-163.

ARAUJO, Nadya. Home and Market, Love and Work, Nature and Profession: Controversies regarding the commodification of care work. cadernos pagu (46), Campinas-SP, Núcleo de Estudos de GêneroPagu/Unicamp, 2016, pp.59-77.

Blofield, Merike; MADALOzzo, Regina. Conciliação entre Família e Trabalho nas Famílias de Classe Média-Baixa e Baixa em São Paulo. In: Congresso Latino-Americano de Estudos do Trabalho, São Paulo, July 4, 2013.

BLOFIELD, Merike; MARTíNEZ-FRANZONI, Juliana. Maternalism, coresponsibility, and social equity: a typology of work-family policies. Social Politics: International Studies in Gender, State \& Society 22(1), 2014, pp.38-59.

BorREL, Carme et alii. Perceived sexism as a health determinant in Spain. Journal of Womens Health (19), 2010, pp.741-750.

BudgEON, Shelley. The dynamics of gender hegemony: Femininities, masculinities and social change. Sociology 48(2), 2014 pp.317-334.

CASTILla, Emilio J.; BenARD, Stephen. The paradox of meritocracy in organizations. Administrative Science Quarterly (55), 2010, pp.543576.

ChAPPell, Louise; GeORgINA Waylen. Gender and the Hidden Life of Institutions. Public Administration 91(3), 2013, pp.599-615. 
CORDERO, Teresita. Un breve recuento sobre las acciones para la equidad de género en la Universidad de Costa Rica. cadernos pagu (49), Campinas-SP, Núcleo de Estudos de Gênero-Pagu/Unicamp, 2017, e174909.

CORRELL, Shelley J.; BERNARD, Stephen; PAIK, In. Getting a job: Is there a mother-hood penalty? American Journal of Sociology (112), 2007, pp.1297-1338.

EAGLY, Alice H.; KARAU, Steven J. Role congruity theory of prejudice toward female leaders. Psychological Review (109), 2002, pp.573-598.

FRASER, Gloria; OsBORNE, Danny; SIBLEY, Chris. G. "We want you in the workplace, but only in a skirt!" Social Dominance Orientation, genderbased affirmative action and the moderating role of Benevolent Sexism. Sex Roles 73(5-6), 2015, pp.231-244.

GLICK, Peter. BS at work: how benevolent sexism undermines women and justifies backlash. In The Harvard Business School symposium Gender \& Work: Challenging Conventional Wisdom, Boston, MA., 2013.

GLICK, Peter; FISKE, Susan. T. An ambivalent alliance: hostile and benevolent sexism as complementary justifications for gender inequality. American Psychologist (56), 2001 pp.109-118.

GUEDES, Moema de Castro. Heterogeneização da mão-de-obra feminina: o que mudou no período recente? cadernos pagu (47), Campinas-SP, Núcleo de Estudos de Gênero-Pagu/Unicamp, 2016, e164720.

HoOBlen, Jenny. M.; WAYNE, Sandy. J.; LeMmON, Grace. Bosses' perceptions of family - work conflict and women's promotability: Glass ceiling effects. Academy of Management Journal (52), 2009, pp.939957.

JACKMAN, Mary. R. The velvet glove: Paternalism and conflict in gender, class, and race relations. Oakland, CA, University of California Press, 1994.

JUDGE, Timothy A.; CABLE, Daniel. M. When it comes to pay, do the thin win? The effect of weight on pay for men and women. Journal of Applied Psychology (96), 2011, pp.95-112.

KING, Eden B. et alii. Benevolent sexism at work: gender differences in the distribution of challenging developmental experiences. Journal of Management, 38, 2012, pp.1835-1866. 
Manganelli, Anna Maria; BobBio, Andrea; CANOvA, Luigina. Sexism, conservative ideology and attitudes toward women as managers. Psicologia sociale 7(2), 2012, pp.241-260.

MINISTERIO de Empleo y Seguridad Social. Informe Anual 2013 de la Inspección de Trabajo y Seguridad Social. Madrid: Dirección General de la Inspección de Trabajo y Seguridad Social, 2013.

MORGAN, Whitney B. et alii. A field experiment: reducing interpersonal discrimination toward pregnant job applicants. Journal of Applied Psychology (98), 2013, pp.799-809.

MosS-RACUSIN, Corine A. et alii. Science faculty's subtle gender biases favor male students. PNAS 109, 2012, pp.16474-16479.

NoseK, Brian A.; BANAJI, Mahzarin; GreENWALd, Anthony, G. Harvesting implicit group attitudes and beliefs from a demonstration web site. Group Dynamics: Theory, Research, and Practice (6), 2002, pp.101115.

OECD. Employment: Gender wage gap. 2011 [http://stats.oecd.org//Index.aspx?Queryld =54751 - consultado el 22 de mayo de 2017]

OIT. Informe Mundial sobre Salarios 2016/2017 La desigualdad salarial en el lugar de trabajo. Organización Internacional del Trabajo. 2016-17 [http://www.ilo.org/global/research/global-reports/global-wagereport/2016/WCMS 537989/lang--es/index.htm - consultado el 4 de mayo de 2017]

OIT. Informe OIT-Gallup, 2017. Hacia un futuro mejor para las mujeres en el trabajo: la opinión de las mujeres y de los hombres. Organización Internacional del Trabajo. Oficina para España. 2017 [http://www.ilo.org/wcmsp5/groups/public/---europe/---ro-geneva/--ilo-madrid/documents/genericdocument/wcms 548308.pdf consultado el 12 de junio de 2017]

RISMAN, Barbara J. Gender as a social structure: Theory wrestling with activism. Gender \& Society, 18(4) 2004. pp.429-50.

RUDMAN, Laurie A. et alii. Status incongruity and backlash effects: defending the gender hierarchy motivates prejudice against female leaders. Journal of Experimental Social Psychology (48), 2012, pp.165-179. 
SANTAMARÍA, Jose Carlos. La falacia de la libertad de elección familiar de algunas prestaciones para el cuidado de los hijos. Perjuicios sociales, simbólicos y económicos. Asamblea abierta PPiiNA, 2013.

SANTOS, Maria Helena; AMÂNCIO, Lìgia. A (in)justiça relativa da ação afirmativa: A influência do género na controvérsia sobre as quotas baseadas no sexo. Análise Psicológica (28), 2010, pp.43-57

SANTOS, Maria Helena; AMÂNCIO, Lìgia. Género e cidadania: O lento caminho para a paridade. In: ROBERTO, M. et alii (ed.). Research directions in social and organizational psychology vol. 4, Lisbon, Portugal, Colibri, 2011, pp.51-74.

SANTOS, Maria Helena; AMÂNCIO, Lìgia. Gender inequalities in highly qualified professions: a social psychological analysis. Journal of Social and Political Psychology (1), 2016, pp.427-443.

SANTOS, Maria Helena; AmÂNCIO, Lìgia; Roux, Patricia. Numbers do not tell the whole story: Gender and medicine in Portugal. Women's Studies International Forum (53), 2015, pp.73-82.

SAUNDERS, Kendra, J. Women in the Workplace: Feminism's Potential Impact. Handbook on Well-Being of Working Women Springer Netherlands, 2016, pp.565-575.

STAMARSKI, Cailin, S.; SON HING, Leane, S. Gender inequalities in the workplace: the effects of organizational structures, processes, practices, and decision makers' sexism. Frontiers in psychology (6), 2015, pp. 1400 .

WAYLEN, Georgina. Informal Institutions, Institutional Change, and Gender Equality. Political Research Quarterly 67(1), 2014, pp.212223.

WORLD Economic Forum. The global gender gap report 2014 [http://reports.weforum.org/global-gender-gap-report-2014/ consultado el 6 de junio de 2017]. 


\section{Anexo 1: Cuestionario final}

\begin{tabular}{|c|c|c|}
\hline $\mathrm{N}^{\circ}$ & Texto item & C \\
\hline 1 & $\begin{array}{l}\text { El que en algunas profesiones predominen los hombres (mineros, ingenieros, } \\
\text { policías...) y en otras las mujeres (enfermeras, azafatas, secretarias...) se debe a } \\
\text { diferencias naturales entre ambos sexos. }\end{array}$ & \\
\hline 3 & $\begin{array}{l}\text { Las diferencias en el sueldo de hombres y mujeres de la misma categoría y nivel } \\
\text { profesional responden a diferente nivel de productividad o de capacidad de cada } \\
\text { uno/a. }\end{array}$ & \\
\hline 5 & $\begin{array}{l}\text { Las acciones positivas hacia las mujeres (destinarles ayudas específicas, fomentar } \\
\text { su contratación) perjudican a los hombres y a las propias mujeres, porque les } \\
\text { hace parecer inferiores. }\end{array}$ & \\
\hline 6 & $\begin{array}{l}\text { El que haya más mujeres en profesiones de cuidado (enfermeras, maestras...) y } \\
\text { de limpieza se debe a gustos y tendencias naturales de las mujeres. }\end{array}$ & \\
\hline 7 & Hay más hombres empresarios y políticos porque a ellos les interesa más el poder. & \\
\hline 8 & $\begin{array}{l}\text { Un jefe hombre es más objetivo e imparcial y, por tanto, más resolutivo que una } \\
\text { jefa. }\end{array}$ & \\
\hline 9 & $\begin{array}{l}\text { Los hombres llegan más lejos y a mejores puestos en su carrera profesional } \\
\text { porque, por naturaleza, las mujeres son menos competitivas y emprendedoras. }\end{array}$ & \\
\hline 10 & $\begin{array}{l}\text { En el trabajo, los hombres suelen tomar mejores decisiones, porque son más } \\
\text { racionales y objetivos que las mujeres. }\end{array}$ & \\
\hline 11 & $\begin{array}{l}\text { En el espacio público (reuniones laborales, charlas), las mujeres tienen menos } \\
\text { protagonismo, porque les gusta ser más discretas. }\end{array}$ & \\
\hline 12 & $\begin{array}{l}\text { En grupos de trabajo mixtos, se suele escuchar con más detalle cuando interviene } \\
\text { un hombre que una mujer, porque normalmente se explican más claramente. }\end{array}$ & \\
\hline 13 & $\begin{array}{l}\text { Si en una pareja heterosexual hay que encargarse del cuidado de hijos/as u otro } \\
\text { familiar, y alguno de los dos miembros tiene que dejar su trabajo profesional, les } \\
\text { gusta más hacerlo a las mujeres. }\end{array}$ & \\
\hline 14 & $\begin{array}{l}\text { Los hombres son más productivos y rentables para cualquier empresa, ya que las } \\
\text { mujeres suponen muchos más problemas: bajas por maternidad, permisos para } \\
\text { cuidado, ausencias por enfermedades de los hijos/as... }\end{array}$ & \\
\hline 15 & $\begin{array}{l}\text { En estos momentos, las reivindicaciones feministas respecto a condiciones } \\
\text { laborales, abusos de poder etc. han perdido todo su sentido. }\end{array}$ & \\
\hline 16 & $\begin{array}{l}\text { Las bajas de paternidad sólo sirven para que los hombres tengan más vacaciones, } \\
\text { ya que en el momento en que nace el hijo/a el cuidado principal corresponde por } \\
\text { naturaleza a la madre. }\end{array}$ & \\
\hline 18 & $\begin{array}{l}\text { En Educación, las mujeres son mayoritarias en niveles de Infantil porque son más } \\
\text { cariñosas, afectivas, cuidadosas... }\end{array}$ & \\
\hline 20 & $\begin{array}{l}\text { Las mujeres tienden a escoger profesiones y estudios referidos al cuidado (como } \\
\text { psicología, enfermería,...) mientras que los hombres escogen profesiones técnicas } \\
\text { porque es lo que la sociedad espera. }\end{array}$ & \\
\hline 21 & $\begin{array}{l}\text { Las diferencias en la ropa y vestimenta profesionales (ej: faldas en mujeres, uso } \\
\text { de tacón, maquillaje...) se deben a que ellas son más coquetas y les gusta más } \\
\text { cuidar la apariencia. }\end{array}$ & \\
\hline
\end{tabular}

